\title{
Energy Gap in the Aetiology of Body Weight Gain and Obesity: A Challenging Concept with a Complex Evaluation and Pitfalls
}

\author{
Yves Schutz $^{a} \quad$ Nuala M. Byrne ${ }^{b} \quad$ Abdul Dulloo $^{c}$ Andrew P. Hills ${ }^{d}$ \\ a Department of Physiology, Faculty of Medicine, University of Lausanne and University of \\ Fribourg, Lausanne, Switzerland, bInstitute of Health and Biomedical Innovation, School \\ of Human Movement Studies, Queensland University of Technology, Brisbane, Australia, \\ 'Department of Medicine/Physiology, University of Fribourg, Switzerland, ${ }^{\mathrm{d}}$ Centre for \\ Nutrition and Exercise, Mater Research and Centre for Musculoskeletal Research, Mater \\ Mothers Hospital, Griffith Health, Griffith University, Brisbane, Australia
}

\section{Key Words}

Energy gap · Energy imbalance · Food availability · Food balance sheets · Total energy expenditure $\cdot$ Doubly labelled water $\cdot$ Physical activity

\begin{abstract}
The concept of energy gap(s) is useful for understanding the consequence of a small daily, weekly, or monthly positive energy balance and the inconspicuous shift in weight gain ultimately leading to overweight and obesity. Energy gap is a dynamic concept: an initial positive energy gap incurred via an increase in energy intake (or a decrease in physical activity) is not constant, may fade out with time if the initial conditions are maintained, and depends on the 'efficiency' with which the readjustment of the energy imbalance gap occurs with time. The metabolic response to an energy imbalance gap and the magnitude of the energy gap(s) can be estimated by at least two methods, i.e. i) assessment by longitudinal overfeeding studies, imposing (by design) an initial positive energy imbalance gap; ii) retrospective assessment based on epidemiological surveys, whereby the accumulated endogenous energy storage per unit of time is calculated from the change in body weight and body composition. In order to illustrate the difficulty of accurately assessing an energy gap we have used, as an illustrative example, a recent epidemiological study which tracked changes in total energy intake (estimated by gross food availability) and body weight over 3 decades in the US, combined with total energy expenditure prediction from body weight using doubly labelled water data. At the population level, the study attempted to assess the cause of the energy gap purported to be entirely due to increased food intake. Based on an estimate of change in energy intake
\end{abstract}


judged to be more reliable (i.e. in the same study population) and together with calculations of simple energetic indices, our analysis suggests that conclusions about the fundamental causes of obesity development in a population (excess intake vs. low physical activity or both) is clouded by a high level of uncertainty.

(C) 2014 S. Karger GmbH, Freiburg

\section{Introduction}

The 'energy gap' concept, when quantitatively applied to the aetiology of weight gain and obesity, has plagued many investigators over the last decade or more. It needs to be kept in mind that, from a historical perspective, the first utilisation of the term 'energy gap' was in the context of malnutrition (not obesity) and that it was employed in relation to Third World Countries, with McLaren [1] utilising the term 'protein gap' 40 years ago. Since then it has been confirmed that it is was not a 'protein gap' per se in the developing world but rather an 'energy gap', meaning that total food intake was deficient rather than specifically low in protein intake.

There is still little consensus regarding the exact definition of energy gap(s) but most investigators agree that, at the population level, it is a relatively small delta. Furthermore, the magnitude of the energy imbalance gap required to cause obesity and the respective contribution of excess energy intake above the energy requirement in combination with a diminished physical activity remains a key question (see [2] for review).

The purpose of this paper is not to criticize the energy gap concept but to highlight the difficulty of accurately tracking this relatively small component and to clarify the different ways of assessing it. The gap in real life is the difference between two large numbers, i.e. total energy intake minus total energy expenditure (TEE), hence a potential difficulty to assess it. This poses the question whether it can really be measured with sufficient accuracy at the population level to draw conclusions about the aetiological factors of weight gain, i.e. a net increase in energy intake and/or a blunted physical activity energy expenditure.

According to Hill [3], the energy gap concept has been developed 'in an attempt to individualize strategies for weight loss maintenance' so that it was defined as 'the difference between energy requirement before and after weight loss'. The term energy gap was also coined by Hill [4] in order to 'to estimate the change in energy intake and expenditure behaviours required to achieve different body weight outcomes in individuals and populations'.

Hall et al. [5] have published an interesting paper which used a mathematical modelling approach to simulate the dynamic of weight change in a population. They have used the term 'maintenance energy gap' for the US population, which slowly gains weight over decades, as being the magnitude of change of energy intake required to maintain the final increased body weight as compared to the initial weight. They have apparently excluded any changes in physical activity above those related to body weight gain per se. In our opinion, this is not a gap in the strict sense for two reasons: Firstly, it is an obligatory response consecutive to a sustained energy imbalance gap. Therefore, it can be viewed as a 'normal' physiological adaptive response of the body to a dislocation of energy balance of various magnitude and duration. It is the response of the sustained energy imbalance gap and not the cause, i.e., an initial energy imbalance gap will, over time, induce a dynamic phase which ultimately leads to a 'maintenance energy gap'. The difficulty of this terminology is that the same energy imbalance gap could lead to a different maintenance energy gap according to the duration of the stimulus. When epidemiological studies are used, the magnitude of this gap is unknown initially, since it uses retrospective calculations. Secondly, one difficulty when using this concept is that the maintenance energy equilibrium required to calculate the 'maintenance 
Schutz et al.: Energy Gap in the Aetiology of Body Weight Gain and Obesity: A Challenging Concept with a Complex Evaluation and Pitfalls

energy gap' may not be reached at the time of observation. When calculating a maintenance energy gap, are we sure that the dynamic phase is over and is maintained for a prolonged period of time? The profile of body weight gain should clearly tail off. A steady-state situation, during which the initial energy gap is fully offset, is probably fragile. Therefore, in our opinion, the maintenance energy gap could simply be viewed as a 'normal' change in 'energy requirement' due to increased body mass and change in body composition, including a putative change in physical activity energy expenditure. Thus, depending upon the magnitude of changes in the latter factors, it is not surprising that the average energy imbalance gap (30 $\mathrm{kJ} /$ day) calculated by Hall et al. [4] by means of retrospective analysis is substantially smaller $(30 \times)$ than the 'maintenance energy gap' of $900 \mathrm{~kJ} /$ day estimated by the authors. Therefore, the factor time appears to be a key factor.

Taken together, the terminology of the 'energy gap' concept could be more consensual and associated with a term indicating which gap we are talking about: Weight gain might be viewed as a 'positive energy imbalance gap' (e.g. in the case of a net increase in metabolisable energy intake over energy requirement), weight loss as a 'negative energy imbalance gap' (e.g. a decrease in energy intake over energy requirement or a net increase in TEE over energy intake), or as a combination of both.

Finally, in a highly marketed paper [6] which led to many hot debates [7-9], a new term was introduced: the so-called 'energy flux gap'. It is a concept which assumes that total energy intake is identical to TEE, therefore proposing the hypothesis that there is no gap between the two variables and that energy balance is in equilibrium (this paper will be discussed in the second part of this article).

Globally, this diverse interpretation of the energy gap concept underlines the need of a consensual debate in the future in order to clarify this concept as well as to keep a critical view about the methodology used for its quantitative assessement .

\section{Energy Gap Is a Dynamic Concept}

The key issue in energy gap assessment is the accuracy of determining the energy imbalance and the time frame considered to extrapolate a constant gap over years (or decades), wrongly assuming that the system is invariant.

Basically, at least two commonly-used methods in order to quantify the energy gap(s) magnitude on a daily basis or to measure the response of the organism to an energy imbalance gap can be distinguished: retrospective evaluation using epidemiological data versus prospective evaluation using experimental intervention during which the gap is imposed by design. These methods are schematically outlined in figure 1.

The first method has been largely used in the literature and is a theoretical approach based on retrospective epidemiological data. The energy gap can be calculated from the trend in energy intake/expenditure together with the body weight pattern measured at the same time points. An estimate of the body tissue energy storage accumulated over the same period (generally years or decades) is calculated from delta body weight change, using an estimated energy density of weight change (kcal/kg weight change) or, more accurately, a value based on the change in body composition.

It should be noted that a positive energy imbalance gap is not in the strict sense equal to body energy accumulated since there is an additional metabolic cost to store energy in the body (fig. 2). The ratio of the amount stored (expressed in energy terms) to the positive energy imbalance gap constitutes an estimate of the net efficiency of energy utilisation ( $\eta$ ) for the storage of substrates (i.e. protein + fat + glycogen). This value is much below 1.0 (i.e. below an efficiency of $100 \%$ ) and typically ranges from 0.75 to 0.85 , indicating that $15-25 \%$ [3] of 


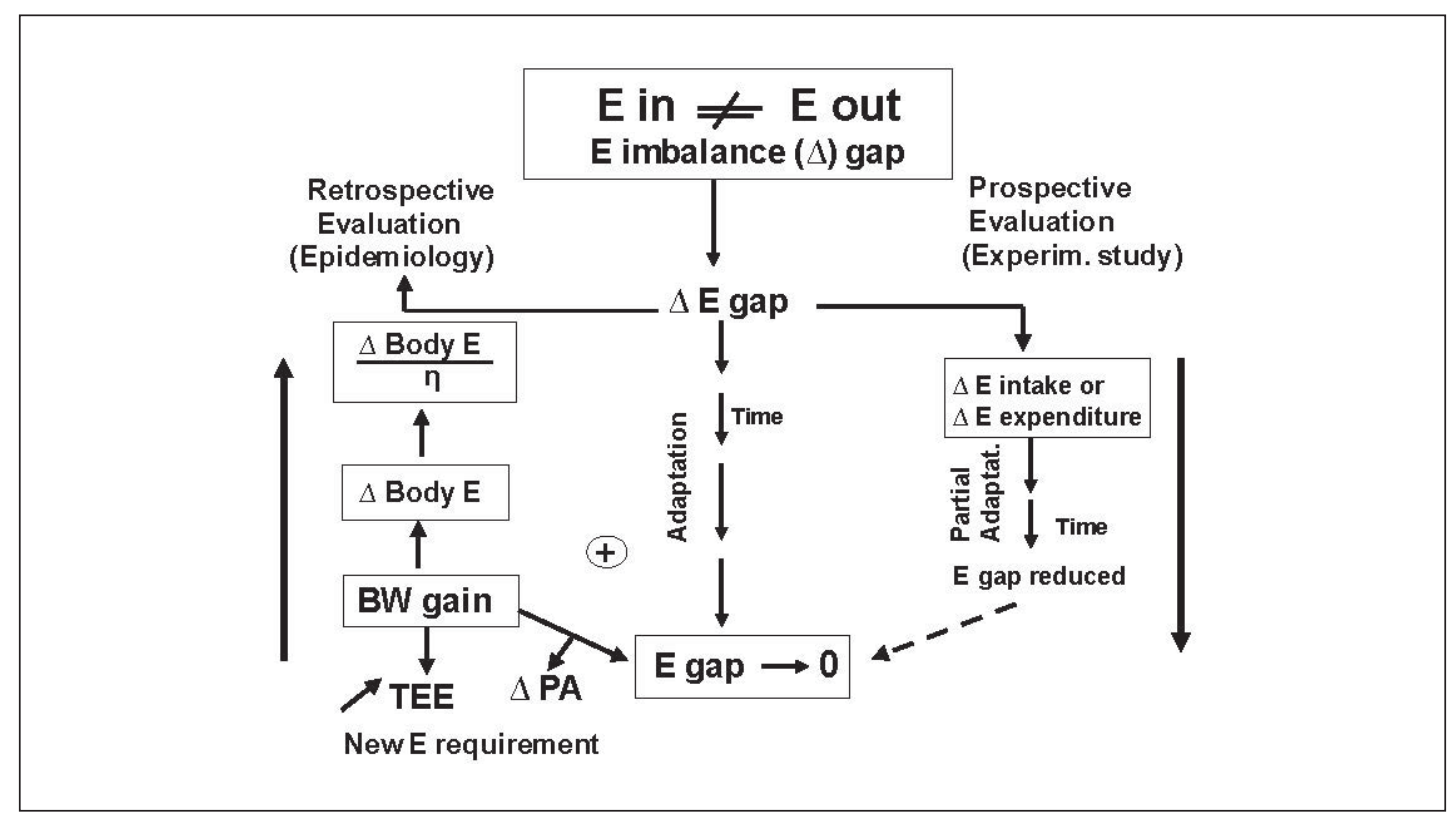

Fig. 1. Two main approaches to evaluate the energy gap either in terms of its metabolic and thermogenic response or in terms of its estimated magnitude: i) prospective assessment by experimental human studies, imposing (by design) an initial positive energy imbalance gap (acute overfeeding studies); ii) retrospective assessment, using epidemiological data from which the theoretical accumulated energy storage (fat and lean tissues) per unit of time during weight gain is calculated based on changes in body weight (and body composition). In the second approach, the net efficiency of energy storage ( $\eta$ ) must also be considered. This can be assessed by the first method. The duration of the evaluation is very different in the two approaches: weeks for the experimental investigations, generally in a limited number of subjects, and years/decades for retrospective epidemiological evaluation in a population or a whole nation.

Fig. 2. Partition of excess energy intake, assessed by energy balance studies, during an overfeeding experiment in humans: surfeit energy occurs at t zero (t0) and is defined as an initial positive energy imbalance gap. The partition between thermogenesis (energy expenditure) and energy storage changes over time (see arrows): A greater fraction of food energy is stored initially than after metabolic adaptations. The resulting increase in tissue mass, in particular fatfree mass, enhances energy expenditure (see fig. 3).

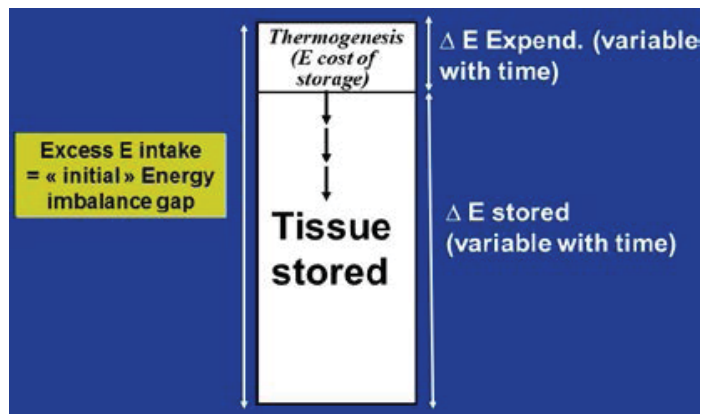

the excess food energy is used to cover the cost of tissue retention as well as the increased macronutrient turnover and other associated processes.

The second method, used to better understand the energetics of a positive imbalance gap, is based on experimental overfeeding studies in humans: A fixed (generally large) excess energy intake above the energy requirement (e.g. a surfeit of $1,000 \mathrm{kcal} /$ day or more) is imposed in an acute study. The relatively short duration of these human studies precludes full metabolic and full physiological adaptations. As a result, although the initial energy imbalance gap tends to decrease with time, a new maintenance energy equilibrium is rarely 
Schutz et al.: Energy Gap in the Aetiology of Body Weight Gain and Obesity: A Challenging Concept with a Complex Evaluation and Pitfalls

Fig. 3. Dynamic response to a step increase in energy (E) intake (positive energy imbalance gap) on the evolution of energy balance. The expected pattern of body weight changes until the new steady state is reached is shown in parallel. The initial energy imbalance gap diminishes progressively over time and slowly vanishes when the new energy equilibrium is reached. The higher resulting body weight is the price to pay to fully offset the initial energy imbalance gap.

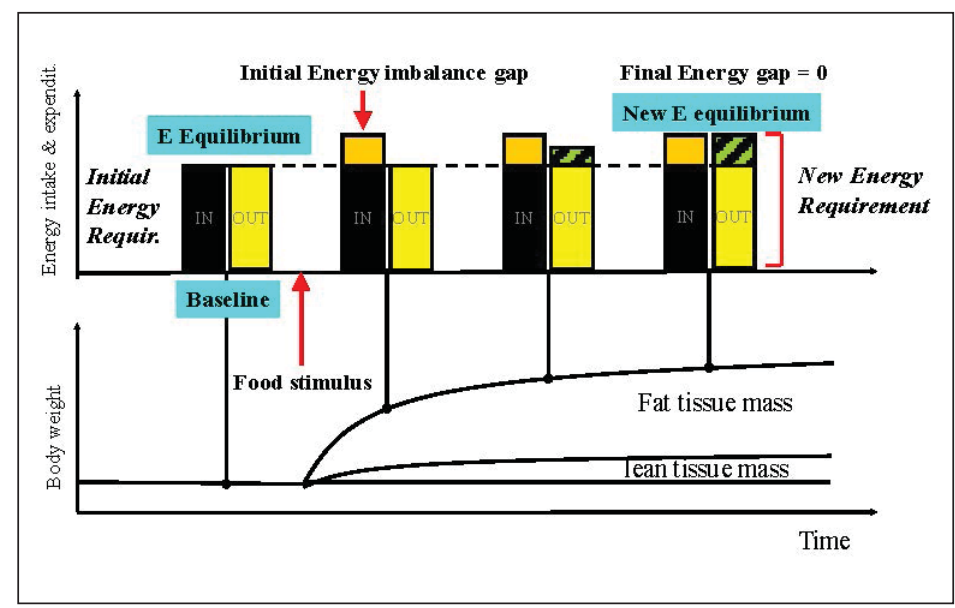

reached in these studies due to the magnitude of energy balance perturbation as well as the limited duration of the experiment. Thanks to these studies, the metabolic energy cost of storing surfeit energy can be measured (e.g. in a respiration chamber) based on the extra thermogenesis incurred by overfeeding macronutrients.

The cost of energy storage is visualized in figure 2, where the partition of surfeit energy intake, assessed by energy balance studies during an overfeeding experiment, is measured at a given time point. In fact, the repartition between increased energy expenditure (by increased thermogenesis) versus increased endogenous energy storage, progressively changes over time. After adaptations, an increase in body mass is the price to pay in order to offset the initial positive energy imbalance gap. The daily storage component diminishes with time, provided the initial conditions remain the same throughout the study and provided the energy intake is fixed and not readjusted progressively to the new energy requirement.

A schematic simplified illustration of the response of a step increase in energy intake (positive energy imbalance gap) on the dynamic of energy balance response as well as the predicted pattern of body weight change is represented graphically in figure 3 . Note that the initial energy imbalance gap does not remain constant but diminishes progressively with time and is ultimately fully counteracted when a new energy balance level is reached thanks to a higher body weight (due primarily to an increased accumulation of fat-free mass tissue and to the additional energy cost of carrying a heavier body weight).

\section{Practical Calculations Using the Epidemiological Approach}

Supposing that, for example, the average (or median) body weight gain is $5 \mathrm{~kg}$ over a 5 -year period, with $4 \mathrm{~kg}$ of adipose tissue stored (about 8,000 kcal/kg), and $1 \mathrm{~kg}$ is lean tissue retained (about 1,000 $\mathrm{kcal} / \mathrm{kg}$ since three quarters are water), the calculated accumulated energy in the body is estimated to be $33,000 \mathrm{kcal}(4 \mathrm{~kg} \times 8,000 \mathrm{kcal} / \mathrm{kg}+1 \mathrm{~kg} \times 1,000$ $\mathrm{kcal} / \mathrm{kg}$ ) spread over 1,825 days, i.e. in theory only $18 \mathrm{kcal}$ of positive energy imbalance gap per day. Other authors have used a constant energy density of weight change averaging 7,700 $\mathrm{kcal} / \mathrm{kg}$ of weight change [10]. However, in order to store $18 \mathrm{kcal}$ per day, we need to eat more than that: Taking an average net efficiency of energy storage of 85\% [3], we need to eat an excess of about $20 \mathrm{kcal}$ daily above the maintenance energy requirement (18 kcal/0.85). In 
conditions of a negative energy imbalance gap, no correction for lower metabolic efficiency is required since the net efficiency of substrate mobilization is close to $100 \%$.

The limitations of the epidemiological approach have already been discussed [3] but, for the following reason, additional correction factors may also be required when applied to a population: If we give a message to a whole population to reduce energy intake by roughly 20 kcal per day (assuming they know what it represents in terms of food), a part of the population may be unable to strictly follow this advice or may not follow this guideline due to psycho-behavioural factors (e.g. systematic poor compliance and volatile nutritional adherence), notwithstanding the fading out of the initial euphoric motivational changes. Assuming that $50 \%$ of the people do not follow the advice and maintain a status quo, an energy reduction of $40 \mathrm{kcal} /$ day among the entire population may theoretically have to be prescribed to get a diminution of $20 \mathrm{kcal} /$ day. This is in view of the bias in the application of the guidelines, particularly in individuals who are overweight or obese, notwithstanding the bias of those subjects who are already in the process of losing weight, as pointed out by Bouchard [11]. This highlights the relative complexity of the epidemiological approach and the wide range of 'corrected' energy imbalance gap which can be generated from the initial value.

\section{Energy Gap in Real Life}

In real life, another difficulty occurs: On a 24 -hour basis, there is a systematic energy imbalance gap of various magnitude since the control of energy intake is not strictly synchronized to energy expenditure on a daily basis. As a result, the energy gap assessed by the two methods presented above is not compatible with real life since positive and negative energy imbalance gap fluctuations spontaneously occur and as these are not necessarily of random nature. Assessment of the energy gap is complex in real life for two reasons: Firstly, the dayto-day energy imbalance may be small in habitual daily living and therefore requires a very accurate method of tracking it, and secondly, the gaps constantly oscillate up and down, generating a cycle of small energy storage followed by small energy mobilization. The oscillations of energy gaps mean that short periods of positive energy gaps of various magnitude (i.e. energy storage) alternate with other periods of negative energy gap (i.e. energy mobilization) so that the calculation of an energetic efficiency value becomes more complicated than in constant initial energy imbalance gap conditions of surfeit energy (experimental study).

\section{What Is the Theoretical Effect of a Small Positive Energy Gap Imbalance over Time in Terms of Weight Gain?}

The magnitude of the energy gap required to produce a given change in body weight at the population level has been the key issue for many investigators [2-7]. An average value can be estimated for a population but factors such as physical activity level (PAL), body composition, and age (growth vs. non-growth) can generate substantially different estimates. Based on a very simple mathematical model, Weinsier et al. [12] made a prediction of body weight gain of lean versus obese adult individuals with an initial positive energy imbalance gap of +100 or $+200 \mathrm{kcal} /$ day (fig. 4) two decades ago. Other more sophisticating modelling approaches have been developed subsequently (see for example [5]). It is noteworthy that the initial energy intake gap occurring at time zero was clamped, thereby explaining the curvilinear, asymptotic response of body weight after many years [12]. Other 
Schutz et al:: Energy Gap in the Aetiology of Body Weight Gain and Obesity: A Challenging Concept with a Complex Evaluation and Pitfalls

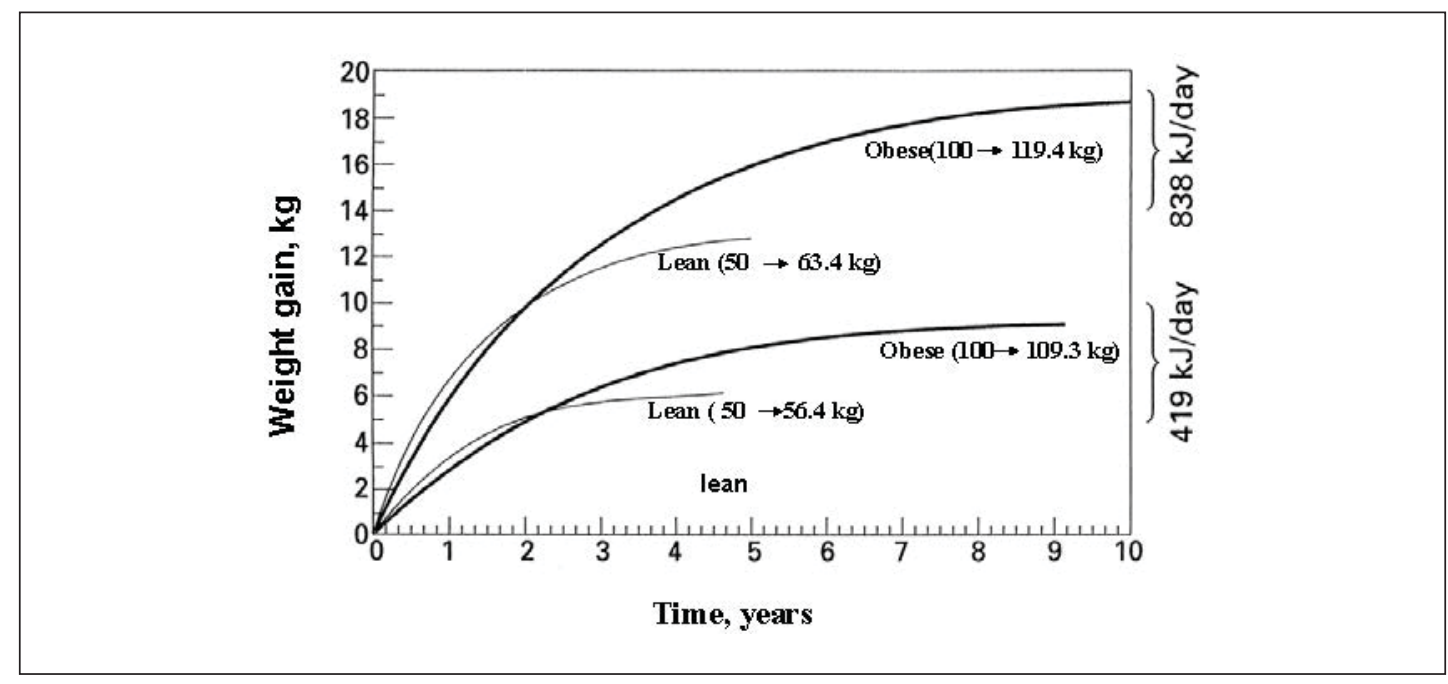

Fig. 4. Prediction of weight gain with a constant initial positive energy imbalance gap of +100 or +200 $\mathrm{kcal} /$ day (419 and $838 \mathrm{~kJ} /$ day, respectively) in lean and obese subjects (based on [12]). It should be noted that the net weight gain is substantially greater in obese (further penalizing it) than in lean individuals for the same initial energy imbalance gap. One important factor explaining this is the initial difference in body composition between the two groups as well as the nature and metabolic activity of tissue stored during weight gain (more fat in obese individuals).

investigators have developed more sophisticated models which arrive at similar conclusions, i.e. the long duration required to offset the initial positive energy gap to reach a steadystate level [2-5].

\section{Analysis of an Epidemiological Study Aimed at Identifying the Cause of an Energy Gap Leading to the Obesity Surge in the USA}

Considering what has been highlighted above, we want to discuss an illustrative example of a highly quoted study [6], which used a semi-epidemiological, semi-physiological, and rough nutritional approach to identify the aetiology of increased obesity at the population level by calculating an 'energy flux gap'. The energy flux was defined as a total energy intake assumed to be equivalent to TEE. Considering the nature of the data used, the issue of identifying the cause of the epidemic of obesity in the US between 1970 and 2000 was a challenging task. Can the rise in obesity be completely explained by an increase in total energy intake in the US population alone, i.e. without considering a concomitant decrease in PAL?

Briefly described, the increment of food availability (or food disappearance rate) was calculated over a 30-year time period in order to predict the weight change at steady-state body weight and to compare it to the weight change actually measured in the population. The food disappearance reflects the gross food availability at the national level. It provides no information on food distribution. Furthermore, the gross availability (transformed into energy value) did not take into account the quantities of food wasted on plates or in the fridge, food spoiled, cooking losses, storage losses at any level, and food not recycled, so it was heavily adjusted using a constant value. It must be noted that a correction for food fed to pets, which is far from negligible due to the extraordinarily increased keeping of cats and dogs in recent decades, is absent, and therefore, it is still inflating the food availability despite correc- 
Schutz et al.: Energy Gap in the Aetiology of Body Weight Gain and Obesity: A Challenging Concept with a Complex Evaluation and Pitfalls

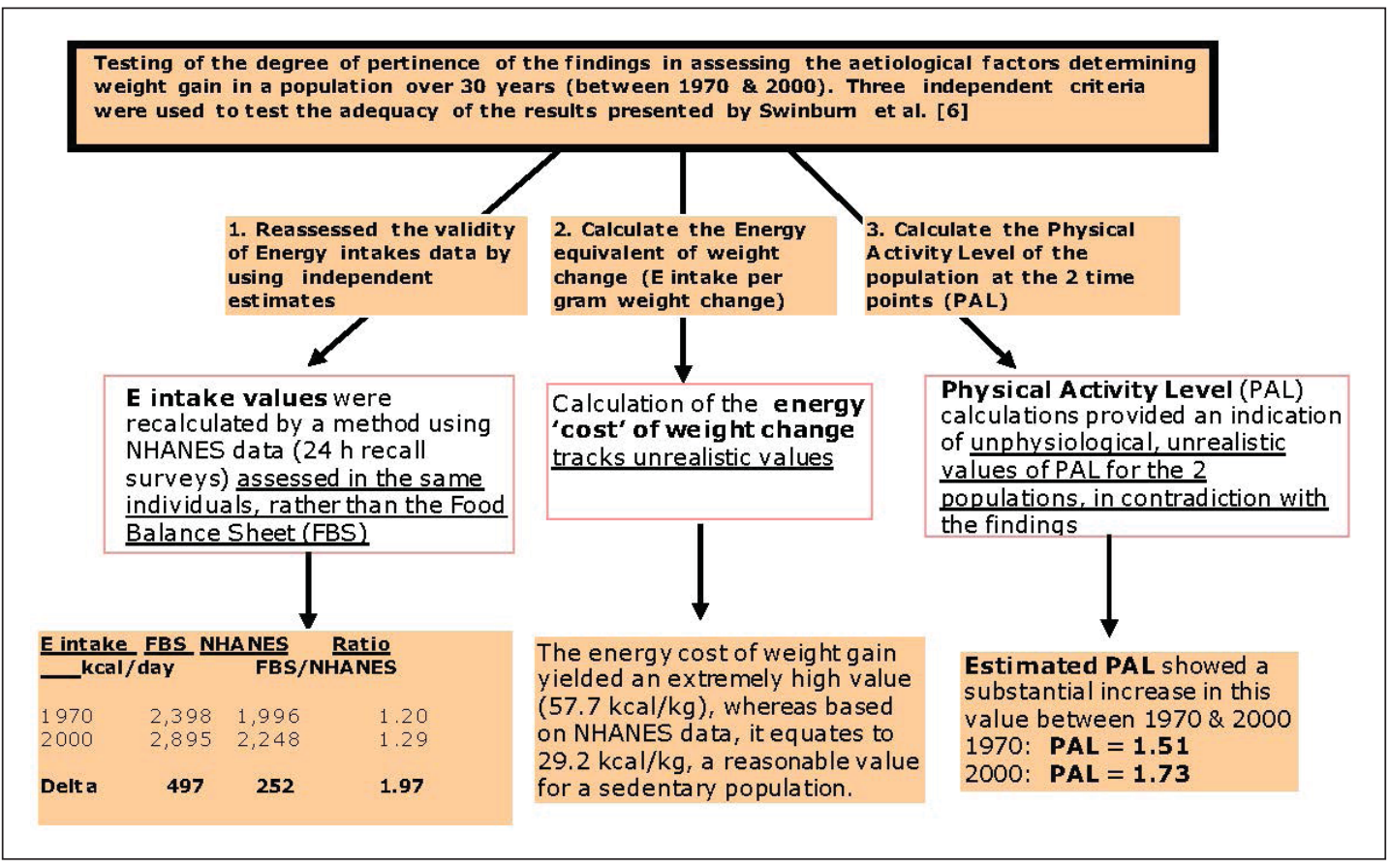

Fig. 5 The three independent approaches used in this review and new data re-analysis to judge the adequacy and the physiological consistency of the food intake and energy gap calculation published by Swinburn et al. [6]. The study aimed at evaluating the cause of obesity in the US between 1970 and 2000. The results show various inconsistencies in the data and some ambiguity, which challenge the conclusion of the paper (see [15] for details).

tions. In addition, food consumed by visitors and food bought by foreigners visiting the US is not fully accounted for and is unlikely to be constant. As a result, the downward adjustment due to total wastage, estimated as a loss of $30 \%$, remains uncertain.

Furthermore, we were surprised that the authors used dichotomous NHANES (National Health and Nutrition Examination Survey) data for body weight, whereas for the food intake data, the NHANES data, obtained from the same subjects, were ignored [13].

TEE was assessed by doubly labelled water (DLW), assumed to be equivalent to total energy intake (the energy flux). A large diversity of datasets from different sources other than the US, namely The Netherlands, New Zealand, and Africa [6], was pooled and used by the authors. The results were based on a comparison of the differences in slopes of 'energy flux' (intake/expenditure) versus body weight at two different points in time 30 years apart. It is noteworthy that one of the factors influencing the slope of TEE (y) on body weight (x) is the change in the PAL of the population [2], i.e. the lower the PAL, the flatter the regression coefficient (the slope).

\section{Limitations and Pitfalls in Data Assessment and Consistency of the above Findings}

We propose here three different approaches, schematized in figure 5, which used independent methodologies and established criteria to estimate the adequacy and physiological consistency of the energy gap results published by Swinburn et al. [6]. 
Schutz et al.: Energy Gap in the Aetiology of Body Weight Gain and Obesity: A Challenging Concept with a Complex Evaluation and Pitfalls

\section{Change of Energy Intake over Three Decades Assessed by an Independent Method}

We have recalculated the energy intakes based on NHANES I (1971-1974) to NHANES IV (1999-2000) studies which used the classical 24-h recall method. The latter method has obvious limitations when compared to other, more accurate techniques used for experimental nutrition studies (dietary inventory method) since it involves self-reporting of food consumption. In our opinion, the 24 -h recall method, which is very practical for epidemiological studies, is judged largely superior to the energy intake calculated from gross food availability of the whole nation, since the data pertained to the same individuals as in the studied population and since there is no need to apportion the food among individuals for non-adults.

Based on NHANES data, a statistically significant increase in average energy intake occurred during the three decades considered [13]. The average energy intake increased from 2,450 to 2,618 kcal/day for men and from 1,542 to 1,877 kcal/day for women. Therefore, the calculated rise between 1971 and 2000 was $168 \mathrm{kcal} /$ day for men and $335 \mathrm{kcal} /$ day for women. The overall increase in food intake was on average $252 \mathrm{kcal} /$ day (fig. 5) which is almost half the value reported by Swinburn et al. [6], calculated from the food supply data (497 kcal/day).

Finally, we must admit that since the study has evaluated a net change in energy intake over time, the magnitude of error will depend upon the reproducibility of the food balance sheet and the degree of constancy of the correction factor.

\section{Energetic Index of Weight Change}

This calculation gives an indication of whether the energy data are out of the physiological, 'expected' window, e.g., when the values are exceptionally low or high based on the world literature. The idea is to assume that a gain in body tissue (i.e. body weight) over decades will necessitate a net increase in energy intake compatible with the energy balance and substrate balance principles as well as consistent with the net efficiency of energy utilisation (fig. 2), assuming extremes of the nature of tissue storage (100\% fat vs. $100 \%$ fat-free mass). Assessment of the energy equivalent of body weight gain ( $8.6 \mathrm{~kg}$ in 30 years) equates to $57.7 \mathrm{kcal} / \mathrm{kg}$ using the data of the authors [6] versus $29.2 \mathrm{kcal} / \mathrm{kg}$ when recalculated based on NHANES energy intake data. This former value suggests that a gain of $1 \mathrm{~kg}$ in body weight results in an increase in energy intake/expenditure of $58 \mathrm{kcal} /$ day, a value which can be considered as rather high and at least not compatible with previous findings.

\section{Change in Physical Activity Level at Two Time Points}

Calculation of the ratio between energy flux (energy intake or expenditure) and resting metabolic rate, i.e. an index of PAL, is useful to identify whether the data reported by the authors [6] corroborate the hypothesis of no change in PAL over a period of 30 years.

It should be noted that the group regression equation developed between body weight and energy flux (to predict the change in body weight) assumes a constant PAL for the whole population. Surprisingly, this is precisely the change in physical activity the authors would like to track. When the resting (not total) energy expenditure of the sample is calculated based on body weight, gender, and age (FAO/WHO equation from 1985, data not reported here), the total energy flux yields a PAL value of 1.51 in 1970 and 1.73 in 2000, suggesting that PAL has increased in 30 years, i.e., an opposite conclusion to the initial hypothesis! Proxy of physical activity used in conjunction with the approach they used would be very useful. Recently, Church et al. [14] tracked changes in daily occupationrelated physical activity in the US over five decades and showed that the continuous decrease in physical activity explained a significant fraction of the increased body weight gain in the US population. 
Table 1. A comparison of the characteristics of food balance sheets (FBS) - calculating the gross food availability - used to evaluate the changes in energy intake from year to year (according to Swinburn et al. [6] as compared to the technique of doubly labelled water (DLW) used to assess total energy expenditure in free living conditions
Schutz et al.: Energy Gap in the Aetiology of Body Weight Gain and Obesity: A Challenging Concept with a Complex Evaluation and Pitfalls

\begin{tabular}{|c|c|c|}
\hline Variables & FBS & DLW \\
\hline Estimation of & energy intake & $\mathrm{EE}$ \\
\hline Level of assessment & total nation & individuals (groups) \\
\hline Sample size & total coverage & sample ( $\mathrm{n}=$ hundreds) \\
\hline Accuracy & very poor & good \\
\hline Precision & medium & good \\
\hline Time scale & 1 year & $1-3$ weeks (adults) \\
\hline Correction factor & $\begin{array}{l}\text { huge, not constant } \\
(30-40 \%)\end{array}$ & acceptable \\
\hline Utilisation & $\begin{array}{l}\text { nutrition policy and } \\
\text { planning, food trend, } \\
\text { international } \\
\text { comparisons etc. }\end{array}$ & $\begin{array}{l}\text { total } \mathrm{EE} \text {, energy } \\
\text { requirements, derivation } \\
\text { of PAL (total EE/ } \\
\text { resting EE) }\end{array}$ \\
\hline
\end{tabular}

$\mathrm{EE}=$ Energy expenditure; $\mathrm{PAL}=$ physical activity level.

\section{Conclusions}

In summary, the utilisation of a mixture of data from various sources and origin in the study published by Swinburn et al. [6] may lead to uncertain and questionable conclusions about the cause of overall weight gain of an adult population, as this cause is virtually impossible to identify accurately due to the large errors involved in the methodology employed [15].

For energy intake assessment, gross national food availability data (food balance sheet), heavily corrected for various food losses (assumed to be constant over time) and selectively partitioned to adulthood, is questionable. For TEE, predicted value from pooling DLW data derived from other non-US heterogeneous populations, using regression analysis characterized by a huge residual variance, could be challenged in terms of accuracy. As summarized in table 1, where the food balance sheet characteristics are compared to the DLW technique, the former method is mostly suitable in the field of agriculture and for food planning, food policy, and economic purposes. In our opinion, it is certainly not adequate for a combination with objective physiological methods. It needs to be noted that a subsequent paper using the same methodological basis, but analysing the situation in UK, reached inconsistent conclusions than that observed in the US, since a gender effect was observed [16].

The identification of the exact causes for the rise in obesity in a population (increased intake only or decreased physical activity or both) has plagued many authorities in the obesity field over the last decade $[2-5,7-9,11]$. The difficulties in determining what is driving the energy gap in obese individuals remain challenging. The detailed analysis of the arguments has been well reviewed by Sørensen [17].

The issue is perhaps more complex than initially anticipated. It requires prospective, longitudinal measurements based on objective, accurate, and precise nutritional and physiological methods, which are generally difficult to use in a large-scale epidemiological study.

\section{Disclosure Statement}

The authors have no conflicts of interest to declare. 
Schutz et al.: Energy Gap in the Aetiology of Body Weight Gain and Obesity: A Challenging Concept with a Complex Evaluation and Pitfalls

\section{References}

1 McLaren DS: The protein gap. Nature 1974;258:113-117.

-2 Millward DJ: Energy balance and obesity: a UK perspective on the gluttony v. sloth debate. Nutr Res Rev 2013; 26:1-21.

-3 Hill JO: Understanding and addressing the epidemic of obesity: an energy balance perspective. Endocr Rev 2006 Dec;27:750-761.

4 Hill JO, Peters JC, Wyatt HR: Using the energy gap to address obesity: a commentary. J Am Diet Assoc 2009; 109:1848-1853.

5 Hall KD, Sacks G, Chandramohan D, Chow CC, Wang YC, Gortmaker SL, Swinburn BA: Quantification of the effect of energy imbalance on body weight. Lancet 2011;378:826-837.

6 Swinburn BA, Sacks G, Ravussin E: Increased food energy supply is more than sufficient to explain the US epidemic of obesity. Am J Clin Nutr 2009;90:1453-1456.

$>7$ Millward DJ: Energy expenditure and the obesity epidemic. Am J Clin Nutr 2010;91:1801-1802; author reply 1802-1804.

-8 Hall KD, Chow CC: Estimating the quantitative relation between food energy intake and changes in body weight. Am J Clin Nutr 2010;91:816; author reply.

-9 Heymsfield SB: How large is the energy gap that accounts for the obesity epidemic? Am J Clin Nutr 2009;89: 1717-1718.

10 Stroebele N, Hill JO, Willich SN: Identifying the energy gap in the German population using results from representative national health surveys (1985-2002).Public Health Nutr 2011;14:44-48.

11 Bouchard C: The magnitude of the energy imbalance in obesity is generally underestimated. Int J Obes 2008; 32:879-880.

12 Weinsier RL, Bracco D, Schutz Y: Predicted effects of small decreases in energy expenditure on weight gain in adult women. Int J Obes Relat Metab Disord 1993;17:693-670.

13 Wright JD, Kennedy-Stephenson J, Wang CY, McDowell MA, Johnson CL: Trends in intake of energy and macronutrients - United States, 1971-2000. JAMA 2004;291:1193-1194.

14 Church TS, Thomas DM, Tudor-Locke C, Katzmarzyk PT, Earnest CP, Rodarte RQ, Martin CK, Blair SN, Bouchard C: Trends over 5 decades in U.S. occupation-related physical activity and their associations with obesity. PLoS One 2011;6:e19657.

15 Dulloo AG, Jacquet J, Montani JP, Schutz Y: Adaptive thermogenesis in human body weight regulation: more of a concept than a measurable entity? Obes Rev 2012;13(suppl 2):105-121.

16 Scarborough P, Burg MR, Foster C, Swinburn BA, Sacks G, Rayner M, Webster P, Allender S: Increased energy intake entirely accounts for increase in body weight in women but not in men in the UK between 1986 and 2000. Br J Nutr 2010;105:1-6.

17 Sørensen TI: Conference on 'Multidisciplinary approaches to nutritional problems'. Symposium on 'Diabetes and health'. Challenges in the study of causation of obesity. Proc Nutr Soc 2009;68:43-54. 